\title{
Issues Affecting the Synthetic Scalability of Ternary Metal Ferrite Nanoparticles
}

\author{
Lauren Morrow ${ }^{1}$ and Andrew R. Barron ${ }^{1,2,3}$ \\ ${ }^{1}$ Department of Chemistry, Rice University, Houston, TX 77005, USA \\ ${ }^{2}$ Department of Materials Science and Nanoengineering, Rice University, Houston, TX 77005, USA \\ ${ }^{3}$ Energy Safety Research Institute, College of Engineering, Swansea University, Singleton Park, Swansea SA2 8PP, UK
}

Correspondence should be addressed to Andrew R. Barron; arb@rice.edu

Received 30 March 2015; Accepted 20 May 2015

Academic Editor: Tapas Sen

Copyright (C) 2015 L. Morrow and A. R. Barron. This is an open access article distributed under the Creative Commons Attribution License, which permits unrestricted use, distribution, and reproduction in any medium, provided the original work is properly cited.

\begin{abstract}
Ternary $\mathrm{Mn}$ - $\mathrm{Zn}$ ferrite $\left(\mathrm{Mn}_{x} \mathrm{Zn}_{1-x} \mathrm{Fe}_{2} \mathrm{O}_{4}\right)$ nanoparticles (NPs) have been prepared by the thermal decomposition of an oleate complex, sodium dodecylbenzenesulfonate (SDBS) mediated hydrazine decomposition of the chloride salts, and triethylene glycol (TREG) mediated thermal decomposition of the metal acetylacetonates. Only the first method was found to facilitate the synthesis of uniform, isolable NPs with the correct $\mathrm{Mn}: \mathrm{Zn}$ ratio $(0.7: 0.3)$ as characterized by small angle X-ray scattering (SAXS), transmission electron microscopy (TEM), and inductively coupled plasma-optical emission spectroscopy (ICP-OES). Scaling allowed for retention of the composition and size; however, attempts to prepare Zn-rich ferrites did not result in NP formation. Thermogravimetric analysis (TGA) indicated that the incomplete decomposition of the metal-oleate complexes prior to NP nucleation for $\mathrm{Zn}$-rich compositions is the cause.
\end{abstract}

\section{Introduction}

One of the major drawbacks to using nanoparticles in an industrial setting is the lack of commercial scale syntheses for the vast majority of nanoparticles. The syntheses that do exist are often limited to a few selected types of nanoparticles and only use a few different synthetic protocols, despite the fact that the syntheses used may not be the best method to use to make nanoparticles with specific properties $[1,2]$. It has been shown that different syntheses can lead to vastly different properties, even if the nanoparticles appear the same or very similar in terms of size and shape $[1,3-5]$. There are a few reasons as to why there are only a few types of syntheses used in industry: cost of raw materials, quality control, product yield, safety of synthetic method, waste disposal, and environmental concerns. These are just a few factors to keep in mind when determining the efficacy of using a particular synthetic method [6]. However, where the $\mathrm{NP}$ in question is a ternary oxide, there is also an issue of compositional control, that is, $\mathrm{M}^{1}: \mathrm{M}^{2}: \mathrm{M}^{3}$ ratio [7].
We have previously investigated the precursor composition (i.e., ratio of individual metal reagents) on the isolated $\mathrm{NP}$ composition, but the issues associated with changes in scale were not addressed. In this regard we have an interest in the scalability of a class of $(\mathrm{Mn}-\mathrm{Zn}-\mathrm{Fe}-\mathrm{O})$ metal oxide (ferrite) nanoparticles (NPs) as an alternative to nanomagnetite (nMag) as a potential down-hole sensor for reservoir imaging in the oil and gas industry. Despite the success of nMag for biological imaging applications [8-10] and the nontoxic nature of nMag, which would have advantages on environmental grounds [1], we have shown that for a typical shale reservoir composition the quantity of nMag needed for contrast with the rock and proppant could be as much as $242,000 \mathrm{~kg}$ nMag per well [11]. We have shown that $\mathrm{Mn}-\mathrm{Zn}$ ferrite $\left(\mathrm{Mn}_{x} \mathrm{Zn}_{1-x} \mathrm{Fe}_{2} \mathrm{O}_{4}\right)$ NPs have an almost order of magnitude increase in mass susceptibility $\left(\chi_{\text {mass }}\right)$ and thus a greater potential signal-to-noise over the background reservoir rock.

We have investigated three previously reported scalable syntheses of nMag: thermal decomposition of an oleate 
complex [12], sodium dodecylbenzenesulfonate (SDBS) mediated reaction of the metal halides [13], and the triethylene glycol (TREG) mediated reaction of the metal acetylacetonates [14]. Once a methodology was found to be suitable for MnZn ferrite NPs scalability was investigated to determine the synthesis that did not change the composition, size, or shape of the NPs. These results are presented herein.

\section{Materials and Methods}

2.1. Materials. $\mathrm{FeCl}_{3} \cdot 6 \mathrm{H}_{2} \mathrm{O}$ (97\%), $\mathrm{MnCl}_{2} \cdot 4 \mathrm{H}_{2} \mathrm{O}$ (99.9\%), $\mathrm{Zn}\left(\mathrm{NO}_{3}\right)_{2} \cdot 6 \mathrm{H}_{2} \mathrm{O}$ (98\%), $\mathrm{Fe}(\mathrm{acac})_{3} \quad(97 \%), \mathrm{Mn}(\mathrm{acac})_{2}$, $\mathrm{Zn}(\mathrm{acac})_{2} \cdot x \mathrm{H}_{2} \mathrm{O}$ hydrate (powder), and $\mathrm{Fe}\left(\mathrm{NO}_{3}\right)_{3} \cdot 9 \mathrm{H}_{2} \mathrm{O}(98 \%)$ were obtained from Sigma-Aldrich and were used without further purification. Oleic acid $\left(\mathrm{CH}_{3}\left(\mathrm{CH}_{2}\right)_{7} \mathrm{CH}=\mathrm{CH}\left(\mathrm{CH}_{2}\right)_{7} \mathrm{CO}_{2} \mathrm{H}\right.$, 90\%), 1-octadecene $\left(\mathrm{CH}_{3}\left(\mathrm{CH}_{2}\right){ }_{15} \mathrm{CH}=\mathrm{CH}_{2}, 90 \%\right)$, sodium dodecylbenzenesulfonate (SDBS, $\mathrm{C}_{12} \mathrm{H}_{25} \mathrm{C}_{6} \mathrm{H}_{4} \mathrm{SO}_{3} \mathrm{Na}$, technical grade), xylenes ( $\geq 98.5+$ ethylbenzene basis), hydrazine solution (35wt.\% in $\mathrm{H}_{2} \mathrm{O}$ ), triethylene glycol (TREG, $\left.\mathrm{HOC}_{2} \mathrm{H}_{4} \mathrm{OC}_{2} \mathrm{H}_{4} \mathrm{OC}_{2} \mathrm{H}_{4} \mathrm{OH}, 99 \%\right)$, HPLC-grade $\mathrm{H}_{2} \mathrm{O}$ (CHROMASOLV Plus for HPLC), and hexanes (mixture of isomers) were purchased from Sigma-Aldrich and used as received. Ethyl acetate was purchased from EMD Millipore and used as received. Sodium oleate was purchased from TCI Chemicals and was used as received. Ethanol (200 proof) was purchased from Decon Laboratories Inc. and was used as received.

2.2. Characterization. Size determination of the nanoparticles was achieved by small angle X-ray scattering (SAXS) using a Rigaku SmartLab X-Ray diffractometer using a Cu$\mathrm{K}_{\alpha}$ radiation source. Samples were prepared by sealing a concentrated nanoparticle solution in hexanes into a $1 \mathrm{~mm}$ "Glass Number 50 Capillary" tube (Hampton Research Inc.) and the data was resolved using Rigaku's NANO-solver. Inductively coupled plasma-optical emission spectroscopy (ICP-OES) measurements were obtained on a Perkin Elmer Optima 4300DV. Samples were prepared by digesting $0.5 \mathrm{~mL}$ of concentrated nanoparticle solution in $9.5 \mathrm{~mL}$ of concentrated nitric acid. An aliquot of the digested solution $(0.5 \mathrm{~mL})$ was diluted into $9.5 \mathrm{~mL}$ of NANOpure water. ICP standards were obtained from Inorganic Ventures and diluted using NANOpure water. Images were obtained using a JEOL 2010 Transmission Electron Microscope (TEM) on Ultrathin Carbon Type-A support film (Ted Pella). Thermogravimetric analysis (TGA) was conducted on a SDT Q600 under air atmosphere at $50 \mathrm{~mL} / \mathrm{min}$. Samples were placed in a puck (5$10 \mathrm{mg}$ ) and were heated to $600^{\circ} \mathrm{C}$ at a rate of $5^{\circ} \mathrm{C} / \mathrm{min}$.

2.3. Oleate Complex Reaction. Synthesis was based upon a literature procedure [12]. Ethanol, HLPC-grade $\mathrm{H}_{2} \mathrm{O}$, and hexanes were added to a mixture of metal salt(s) and sodium oleate. In the smaller scale reaction, metal salt(s) $(4 \mathrm{mmol}$ total) and sodium acetate $(12 \mathrm{mmol})$ were mixed with ethanol (80 mL), HPLC-grade $\mathrm{H}_{2} \mathrm{O}(3.33 \mathrm{~mol})$, and hexanes $(140 \mathrm{~mL})$. If $\mathrm{Mn}-\mathrm{Zn}$ ferrite nanoparticles were being synthesized, the combination of all of the metal salts had to add up to the total metal salt quantity specified. The solution was placed into a round bottom flask with a stir bar and thermometer and dissolved via stirring under argon with no heat. Once dissolved, the solution was heated to $70^{\circ} \mathrm{C}$ and held for four hours. This creates the metal-oleate complex. After four hours, the solution was cooled to room temperature and washed three times with HPLC-grade $\mathrm{H}_{2} \mathrm{O}$ in a separation funnel. The top organic layer was collected into a preweighed crystallization dish and then the hexanes were evaporated. Once dry, the dish and product were weighed in order to calculate quantity of product. To the product, oleic acid and 1-octadecene were added (smaller scale reaction used $2 \mathrm{mmol}$ oleic acid and $0.079 \mathrm{~mol} 1$-octadecene). The product was dissolved and then transferred into a second round bottom flask. The solution was heated to reflux under an atmosphere of argon and held at reflux for 30 minutes. After that, the solution was cooled to room temperature and then precipitated with ethanol $(50 \mathrm{~mL})$. The solution was centrifuged and washed twice more before being allowed to air-dry. The nanoparticles were then suspended in hexanes.

2.4. SDBS Reaction. Synthesis was based upon a literature procedure [13]. A solution of SDBS $(10 \mathrm{mmol})$ and xylenes $(30 \mathrm{~mL})$ was sonicated in a bath sonicator for a minute. Meanwhile, $\mathrm{FeCl}_{2} \cdot 4 \mathrm{H}_{2} \mathrm{O}(1 \mathrm{mmol}), \mathrm{Fe}\left(\mathrm{NO}_{3}\right)_{2} \cdot 9 \mathrm{H}_{2} \mathrm{O}$ (2 mmol), $\mathrm{MnCl}_{2} \cdot 4 \mathrm{H}_{2} \mathrm{O}(1.05 \mathrm{mmol})$, and $\mathrm{Zn}\left(\mathrm{NO}_{3}\right)_{2} \cdot 6 \mathrm{H}_{2} \mathrm{O}$ $(0.45 \mathrm{mmol})$ were added to ethanol $(1.5 \mathrm{~mL})$ before being added to the SDBS solution in a round bottom flask under vigorous stirring. The resulting solution was stirred for twelve hours at room temperature and under normal atmosphere. At the end of twelve hours, the solution was put under an argon atmosphere and heated to $90^{\circ} \mathrm{C}$. Once heated, hydrazine solution $(5 \mathrm{mmol})$ was added. The solution was then refluxed for five hours. Once refluxing was complete, the solution was cooled to room temperature and precipitated with ethanol. The solution was then centrifuged and washed several times before being air-dried. Once dry, the nanoparticles were suspended in hexanes.

2.5. TREG Reaction. Synthesis was based upon a literature procedure [14]. A mixture of the appropriate metal acetylacetonates $(2 \mathrm{mmol})$ was put into a round bottom flask and triethylene glycol $(25 \mathrm{~mL})$ was added. A ratio of $0.7: 0.3$ $\mathrm{Mn}: \mathrm{Zn}$ was used, with there being twice as much iron as manganese and zinc combined. The solution was put under an argon atmosphere and slowly heated to $180^{\circ} \mathrm{C}$ and then held for 30 minutes. The solution was then heated to reflux and held for 30 minutes before being cooled to room temperature. Ethanol $(10 \mathrm{~mL})$ and ethyl acetate $(20 \mathrm{~mL})$ were added. A handheld magnet was then used to separate the nanoparticles from solution. The nanoparticles were washed three more times with a combination of ethanol and ethyl acetate before being air-dried. Once dried, the nanoparticles were suspended in a polar solvent.

\section{Results and Discussion}

As noted in the Introduction, a key driving force for the development of the nanoparticles as tracers is the need to be able to produce the nanoparticles to scale. Our prior synthesis of $\mathrm{Mn}-\mathrm{Zn}$ ferrite NPs was through the thermal 


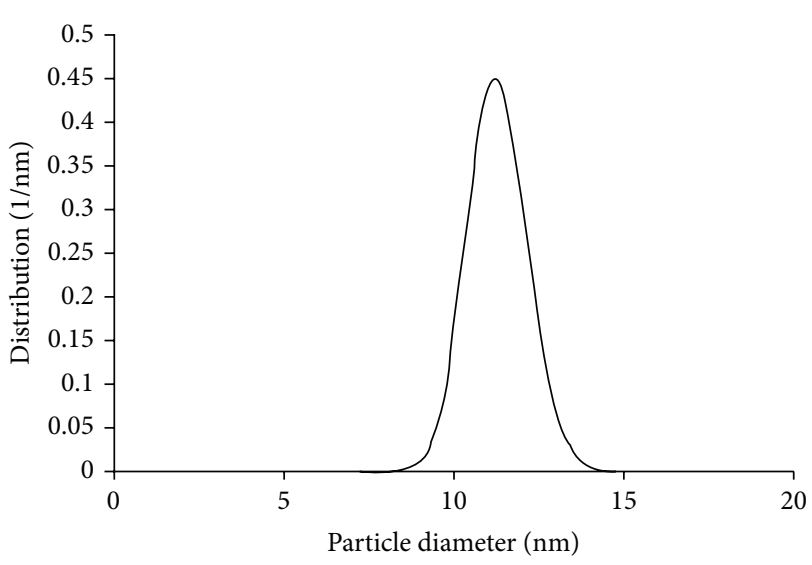

(a)

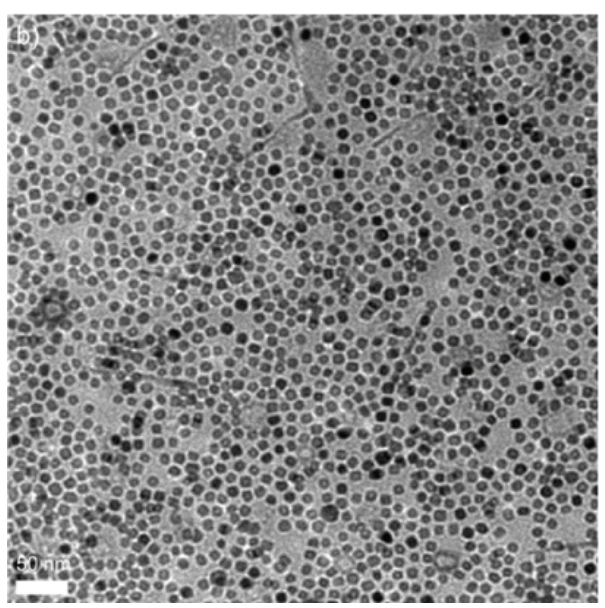

(b)

Figure 1: The size and shape control of Mn-Zn ferrite nanoparticles synthesized through a metal-oleate complex reaction as determined by (a) SAXS and (b) TEM (representative image shown). The scale bar (bottom left) is $50 \mathrm{~nm}$.

decomposition of the metal acetylacetonates [7] and this was used as a benchmark with regard to yield, composition, and cost. To that end, three scalable reactions were investigated and compared to see which one produces nanoparticles with the desired characteristics and with the highest level of control over size and shape, best size dispersion, lack of aggregation, and compositional control with the least cost [12-14]. In order to be able to determine the size and shape dispersion, as well as compositional accuracy, small angle $\mathrm{X}$-ray scattering (SAXS), transmission electron microscopy (TEM), and inductively coupled plasma-optical emission spectroscopy (ICP-OES) were conducted.

3.1. Synthetic Comparisons. The first reaction carried out was a thermal decomposition of an oleate complex [12]. The first nanoparticles to be synthesized with this method were $\mathrm{Mn}$ $\mathrm{Zn}$ ferrite NPs with a composition of $0.7: 0.3 \mathrm{Mn}: \mathrm{Zn}$. The cost of the starting materials is roughly $\$ 5.99$ per gram of product, with a yield of $95 \%$. While this is not cheap, it is less than what it costs to synthesize the same nanoparticles through a thermal decomposition of metal acetylacetonates [7]. Additionally, this was calculated using prices that were not bulk pricing, so it can be reasonably assumed that the price will decrease upon significant scaling.

In order to create nanoparticles of monodispersity and uniform composition, the oleate complex synthesized was a mixture of iron, manganese, and zinc as opposed to mixing each metal-oleate complex separately [15]. When the nanoparticles were analyzed in SAXS (Figure 1(a)), the average particle size was determined as $11.2 \mathrm{~nm} \pm 0.89$, which is in close agreement with the value obtained from TEM measurements $(11.2 \mathrm{~nm} \pm 1.26)$. The size found for our previously reported Mn-Zn ferrite NPs [7] with a composition of $0.7: 0.3 \mathrm{Mn}: \mathrm{Zn}$ was $6.7 \mathrm{~nm} \pm 0.9$ [7], and thus the oleate synthesis appears to result in slightly larger NPs; however, as can be seen from the TEM image in Figure 1(b),

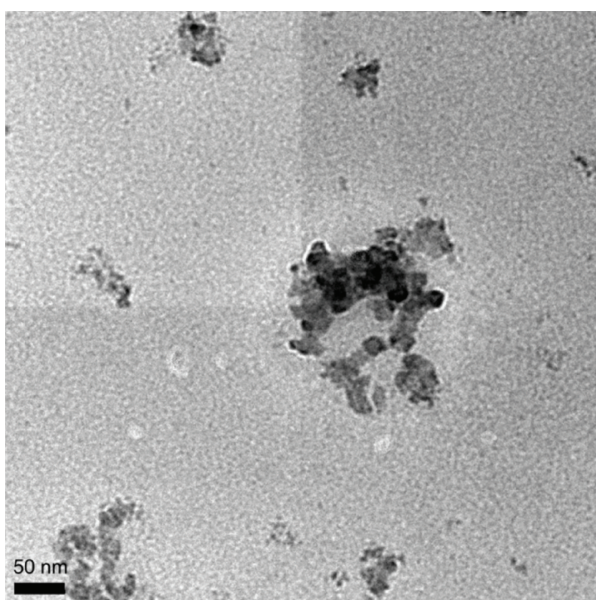

Figure 2: A representative TEM image of the Mn-Zn ferrite NPs synthesized through the SDBS mediated reaction of the metal chloride precursors. The scale bar (bottom left) is $50 \mathrm{~nm}$.

the oleate synthesized NPs are also highly spherical, which, as compared to the original synthesis used to make these nanoparticles, is promising for size and shape control [7].

The second synthesis investigated was a modification of a synthesis utilizing sodium dodecylbenzenesulfonate (SDBS) [13]. This synthesis had significantly less control over the shape and size of the nanoparticles. The morphology seen in TEM shows an aggregated nonsymmetrical product with a large size distribution (Figure 2). Given that SDBS is known to act as a surface-capping agent for NP growth, the lack of size and shape control is more probably due to the different reactivity of the metal salts. An additional issue with the SDBS moderated synthesis is that the nanoparticles would not suspend readily in any solvent. In order to collect SAXS data, a concentrated sample is required and thus we were unable to analyze the SDBS stabilized NPs via SAXS. Furthermore, 


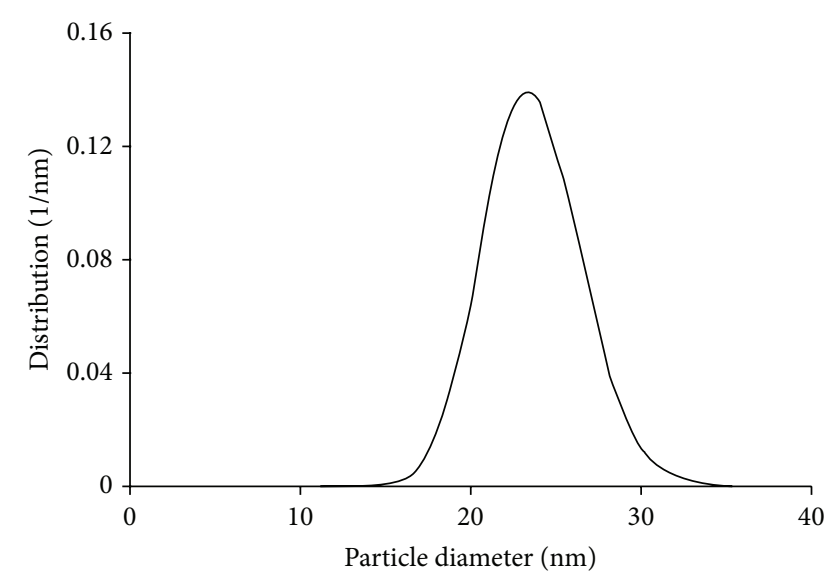

(a)

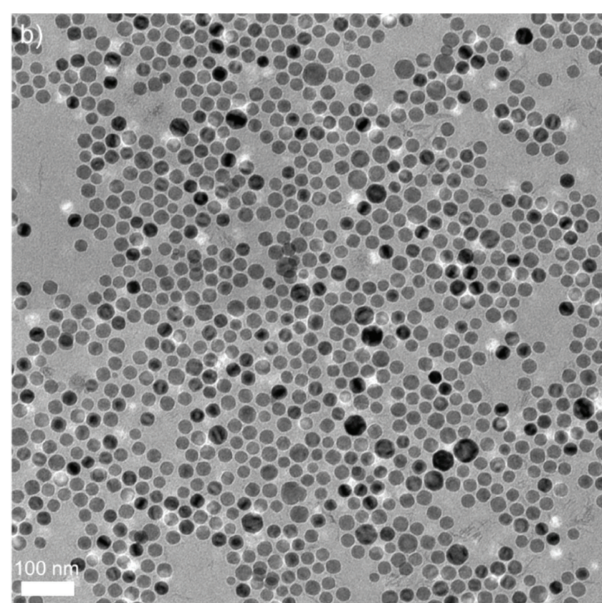

(b)

FIGURE 3: The size and shape control of nMag synthesized through a scaled-up metal-oleate complex reaction as determined by (a) SAXS and (b) TEM (representative image shown). The scale bar (bottom left) is $100 \mathrm{~nm}$.

the synthesis had a very low yield $(<5 \%)$ making it unsuitable for scaling.

The third utilized reaction investigated was the triethylene glycol (TREG) mediated decomposition of the appropriate metal acetylacetonates [14]. While this synthetic method is appropriate for nMag, it preceded so poorly for the Mn- $\mathrm{Zn}$ ferrite system that the product was unable to be suspended in even polar solvents, in contrast to the results reported for nMag [14]. In addition, a range of nonpolar solvents was tried, but the nanoparticles could not be suspended. Due to this, the reaction route was not further pursued. However, we note that part of the issue may be the dramatic differences in the thermal stability of the $\mathrm{M}(\mathrm{acac})_{n}$ complexes. The thermal decomposition of $\mathrm{Fe}(\mathrm{acac})_{3}$ is at $186^{\circ} \mathrm{C}$ [16]; in contrast that of $\mathrm{Mn}(\mathrm{acac})_{2}$ is not until $249^{\circ} \mathrm{C}$ [17], while $\mathrm{Zn}(\mathrm{acac})_{2}$ is at only $130^{\circ} \mathrm{C}$ [18]. Thus, while this route is successful for nMag [14], the high stability of the Mn precursor means that insufficient precursor species are present for La Mer nucleation. Song et al. have shown that issues involving different thermal stability of $\mathrm{M}(\mathrm{acac})_{n}$ species may be overcome through the use of alternative ligands, such as benzoylacetonate [17].

As two of the three potential reactions produced nanoparticles capable of being analyzed further comparisons were made. As seen in Figures 1(b) and 2, the TEM images for both the oleate complex and SDBS reactions show distinct differences in the size, size distribution, and aggregation of the nanoparticles synthesized. If only TEM is consulted, the preferred synthesis to create large quantities of nanoparticles is the oleate complex synthesis. However, it is still unclear whether the nanoparticles from either of the reactions were synthesized with the desired ratios of $\mathrm{Mn}: \mathrm{Zn}$ as compared to the ratios found in the precursors.

Table 1 shows the $\mathrm{Mn}: \mathrm{Zn}$ ratio of the precursors against the ratio of $\mathrm{Mn}: \mathrm{Zn}$ in the NPs as determined by ICP-OES. As can be seen, the oleate complex synthesis highly correlates to the ideal compositional ratio in a similar manner to
TABLE 1: Compositional comparisons between reagent and NP composition as determined by ICP-OES.

\begin{tabular}{lcc}
\hline Synthetic route & Precursor ratio $(\mathrm{Mn}: \mathrm{Zn})$ & $\mathrm{Mn}: \mathrm{Zn}$ ratio in NP \\
\hline Oleate thermolysis & 2.33 & 2.32 \\
$\mathrm{SDBS} / \mathrm{MCl}_{x}$ & 2.33 & 0.95 \\
\hline
\end{tabular}

the thermal decomposition of the metal acetylacetonates [7]. The SDBS synthesis, on the other hand, shows a poor correlation, with a zinc-rich composition making predictable syntheses difficult [7].

Based on results from TEM, SAXS, and ICP-OES, it can be clearly seen that the oleate complex reaction was the ideal synthesis, with high levels of control over size and shape, size distribution, aggregation (or lack thereof), and composition. Thus, all further syntheses were conducted with the oleate complex reaction.

3.2. Scaling Up the Synthesis. For the comparison between the different reactions that have previously been reported to be scalable for nMag [12-14], discussed above, the total amount of nanoparticles per synthesis was up to a few grams. In order to be able to use the nanoparticles as tracers in hydraulically fractured well as intended, significantly more material needs to be synthesized. As an initial test nanoscale magnetite (nMag) was synthesized at a 10x scale to test the feasibility. SAXS analysis of the resulting nMag (Figure 3(a)) shows an average particle size of $24 \mathrm{~nm} \pm 3$. Analysis of the TEM images (Figure 3(b)) shows an average particle size of $27 \mathrm{~nm} \pm 2$, which is in close agreement with SAXS analysis. It should be noted, however, that the NP size is larger than that reported for the smaller scale synthesis of nMag [12] and also that of the Mn- $\mathrm{Zn}$ ferrite nanoparticles (see Figure 1). It is well known, however, that the insertion of cations into the lattice of magnetite changes the size of the synthesized 


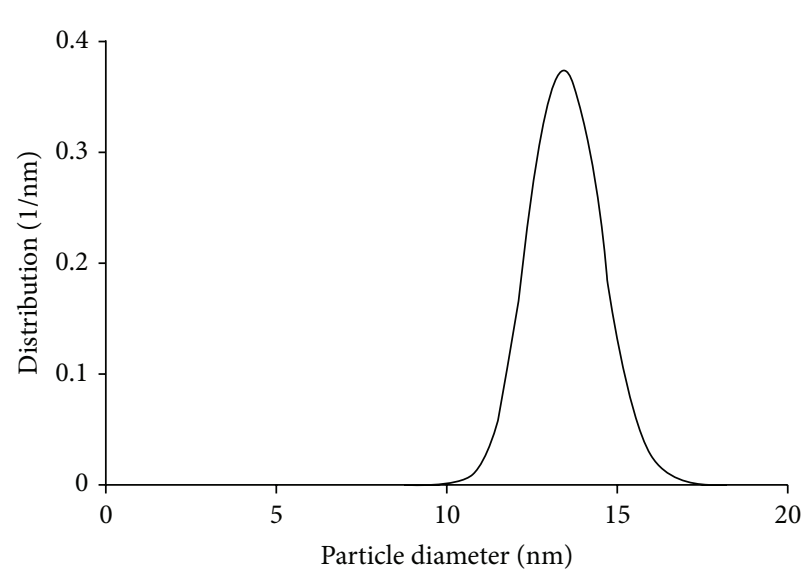

(a)

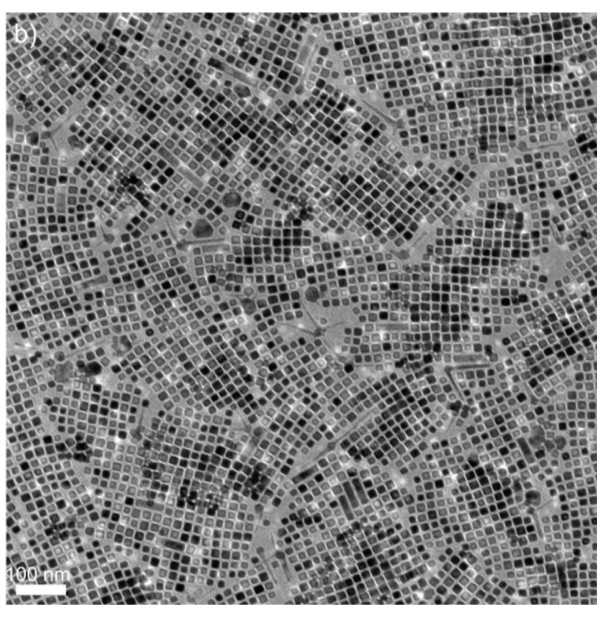

(b)

Figure 4: The size and shape control of Mn-Zn ferrite nanoparticles synthesized through a scaled-up metal-oleate complex reaction as determined by (a) SAXS and (b) TEM (representative image shown). The scale bar (bottom left) is $100 \mathrm{~nm}$.

nanoparticles [19-23]. The difference may be partially due to the said substitution.

Given the successful large-scale synthesis of nMag we attempted a larger scale reaction being run for $\mathrm{Mn}-\mathrm{Zn}$ ferrite nanoparticles. The nanoparticles that resulted from this scaling were analyzed with SAXS, TEM, and ICPOES. Much like the results seen in the smaller syntheses, the sizes obtained from SAXS and TEM (Figure 4) are in close agreement ( $14 \mathrm{~nm} \pm 1$ and $16 \mathrm{~nm} \pm 2$, resp.) and the nanoparticles maintain the small size distribution seen at the lower scale (see above). ICP-OES indicated that the quantity of $\mathrm{Mn}: \mathrm{Zn}$ found in the nanoparticles is the same as that of the precursors, meaning that even as the reaction is scaled the ability of compositional control does not change.

It would appear therefore that the NP size and composition ( $\mathrm{Mn}: \mathrm{Zn}$ ratio) remain essentially unchanged by reaction scale using the oleate synthesis. There is, however, one noticeable difference between the small- and large-scale syntheses: the larger scaled synthesis nanoparticles are of significantly different shape compared to those from the smaller synthesis. As may be seen from Figure 4(b) the NPs are cubic as opposed to spherical (Figure 1(b)) despite having the same precursor ratio of $\mathrm{Mn}: \mathrm{Zn}$. Based upon prior studies we can propose that this cubic structure could be due to impurities in the carboxylate precursor, length of time allowed to age during the growth stage, and/or an excess of fatty acid [15, 24-28]. Irrespective of shape, the ability to scale the synthesis of $\mathrm{Mn}-\mathrm{Zn}$ ferrites has been shown with retention of composition for $\mathrm{Mn}_{0.7} \mathrm{Zn}_{0.3} \mathrm{Fe}_{2} \mathrm{O}_{4}$.

3.3. Relative Oleate Formation and Stability. The success of scaling the reaction for $\mathrm{Mn}-\mathrm{Zn}$ ferrites with a $\mathrm{Mn}: \mathrm{Zn}$ ratio $=0.7: 0.3$ led us to investigate higher $\mathrm{Zn}$ content using the oleate route, because of the higher mass susceptibility $\left(\chi_{\text {mass }}\right)$ observed for $\mathrm{Zn}$-rich materials. Unfortunately, when compositions were attempted with $\mathrm{Mn}: \mathrm{Zn}$ ratio $<1$, no NPs could be isolated.
The highly uniform size and shape of the NPs in the present case are consistent with La Mer nucleation as was observed for nMag $[29,30]$. In the case of nMag it has been proposed that it is the thermal decomposition of iron-oleate complex that generates intermediate species, which seem to act as monomers for the iron oxide nanocrystals. It has been previously shown that the synthesis of metal oxide NPs in a nonaqueous solution occurs via the formation of an aldehyde radical [31]. Furthermore, during growth both oleate complexes and nanocrystals are present [29]. Thus, while Zn, $\mathrm{Mn}$, and $\mathrm{Fe}$ are shown to complex readily with oleate $[5,12$, 32 ], in order to form mixed metal nanoparticles, the oleate complex must have a uniform distribution of the various metals throughout and those metal-oleate complexes must have similar decomposition temperatures [15, 26, 33, 34].

At low concentrations of zinc, nanoparticles are readily synthesized and therefore it can be assumed that the oleate complex is equally mixed and uniform throughout. At higher quantities of zinc, however, nanoparticles do not form from the oleate complex, which suggests that there is some change in the mixture of the oleate complex, preventing the creation of mixed metal oxide nanoparticles. In order to determine whether the oleate complex was forming in a nonuniform manner with higher quantities of zinc, the thermogravimetric analysis (TGA) was investigated for iron-, manganese-, and zinc-oleate complexes, as well as two different ironmanganese-zinc-oleate complexes of varying compositions.

The Fe(III)-, Mn(II)-, and Zn(II)-oleate complexes have TGA curves that are similar to that seen in the literature (Figures 5(a)-5(c)) [5, 12, 32, 35]. The iron(III) complex has two distinct weight loss regimes (Figure 5(a)). The first section starting around $160^{\circ} \mathrm{C}$ correlates to a single oleate ligand being dissociated from the complex, while the second $\left(\right.$ at $300^{\circ} \mathrm{C}$ ) correlates to the remaining two oleate ligands dissociating. Bao et al. have proposed that the middle section, where the slope of the graph flattens, is associated with the dissociation of oleate molecules that are weakly bound or 


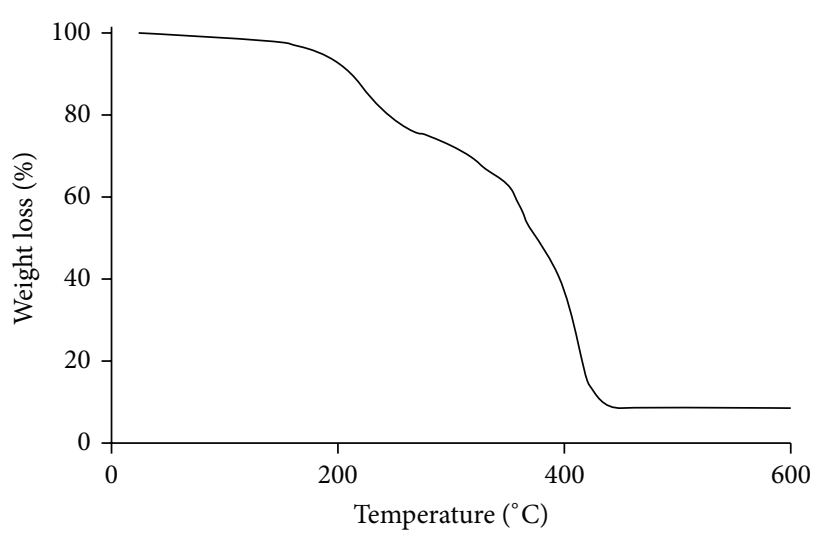

(a)

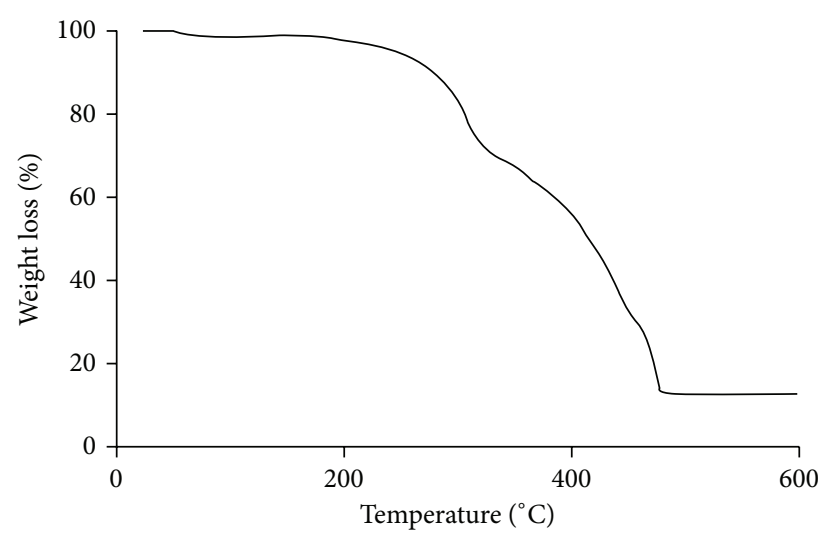

(b)

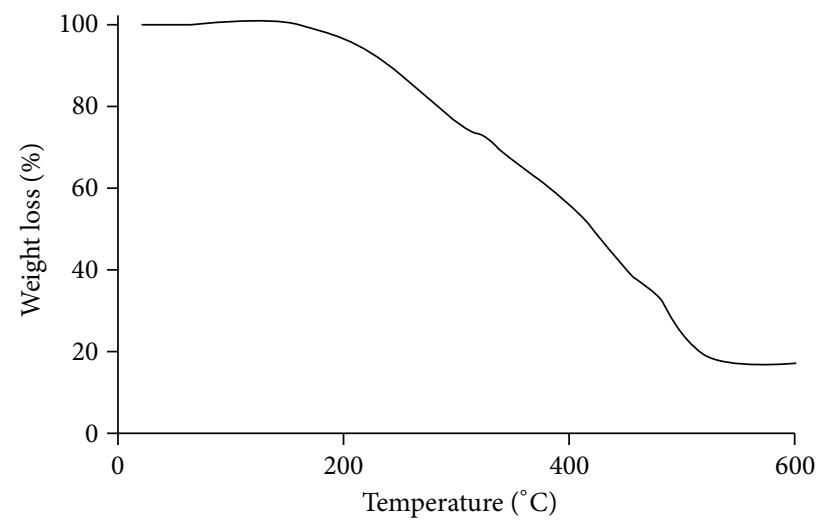

(c)

Figure 5: TGA curves of (a) Fe-oleate complex, (b) Mn-oleate complex, and (c) Zn-oleate complex.

are even free [15]. Manganese(II)-oleate has a similar curve when compared to iron(III)-oleate, but with the temperatures associated with weight loss slightly higher $\left(240^{\circ} \mathrm{C}\right.$ and $400^{\circ} \mathrm{C}$, resp.), as well as slightly steeper rates of weight loss (Figure 5(b)). In contrast, the zinc(II)-oleate exhibits a very different TGA curve when compared to iron and/or manganese analogues. There is only a single continuous weight loss between $150^{\circ} \mathrm{C}$ and $500^{\circ} \mathrm{C}$.

An important piece of information to take away from Figure 5 is the change in rate of weight loss for each oleate occurring at a slightly different temperature. Each change in rate of weight loss has been previously associated with different steps of nanoparticle synthesis $[12,15]$. The slower rates of weight loss at lower temperatures are associated with the prenucleation stage, the subsequent slower rate loss at slightly higher temperatures is associated with the nucleation stage, and the high rates at high temperatures are associated with the growth stage of the nanoparticles. The temperatures associated with these changes are important, as they indicate the temperatures needed in order to be able to trigger the appropriate stage. As different metal-oleates are mixed, these stages and temperatures need to be closely observed; otherwise nanoparticle formation may not occur.

In order to investigate the relative thermal events that control the phases of NP growth, two different ironmanganese-zinc-oleate complexes were synthesized and analyzed by TGA and compared to the individual metaloleate complexes discussed above (Figure 5). Figure 6(a) is the comparison of a $\mathrm{Mn}: \mathrm{Zn}$ mixture of $0.3: 0.7$, that is, the zinc-rich regime where NPs are not formed with the component oleate complexes. Based upon the analysis by Bao et al., the mixed metal-oleate complex has a prenucleation stage between $190^{\circ} \mathrm{C}$ and $270^{\circ} \mathrm{C}$. Both iron- and zincoleate complexes have prenucleation stages that occur around the same temperatures, whereas manganese-oleate complex needs at least $240^{\circ} \mathrm{C}$ in order to begin the prenucleation stage. Thus, prenucleation would be expected to favor iron and zinc. The nucleation stage for the mixed metal-oleate complex begins at $270^{\circ} \mathrm{C}$ but ends by $300^{\circ} \mathrm{C}$. Only iron-oleate complex completes the nucleation phase by that temperature. The formation of nanoparticles occurs at temperatures above $300^{\circ} \mathrm{C}$ for the mixed metal-oleate complexes. While this is also true for the other metal-oleate complexes, if the oleate molecules have not dissociated fully, nanoparticle formation will be halted and the reaction will fail. This is therefore consistent with the observed lack of NPs for Zn-rich Mn-Zn ferrites.

The second of the mixed metal-oleate complexes studied $(\mathrm{Mn}: \mathrm{Zn}$ ratio $=0.7: 0.3)$ is quite different from the first. The curves for the various metal-oleate complexes more closely match the curve for the mixed metal-oleate complex (Figure 6(b)). The prenucleation stage occurs at temperatures lower than $270^{\circ} \mathrm{C}$, which is similar to that seen for 


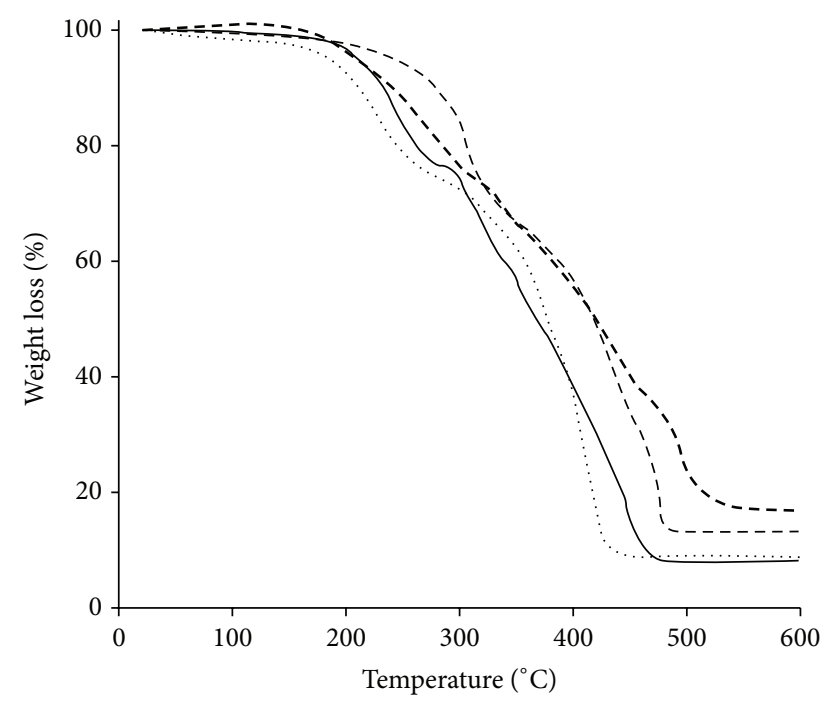

(a)

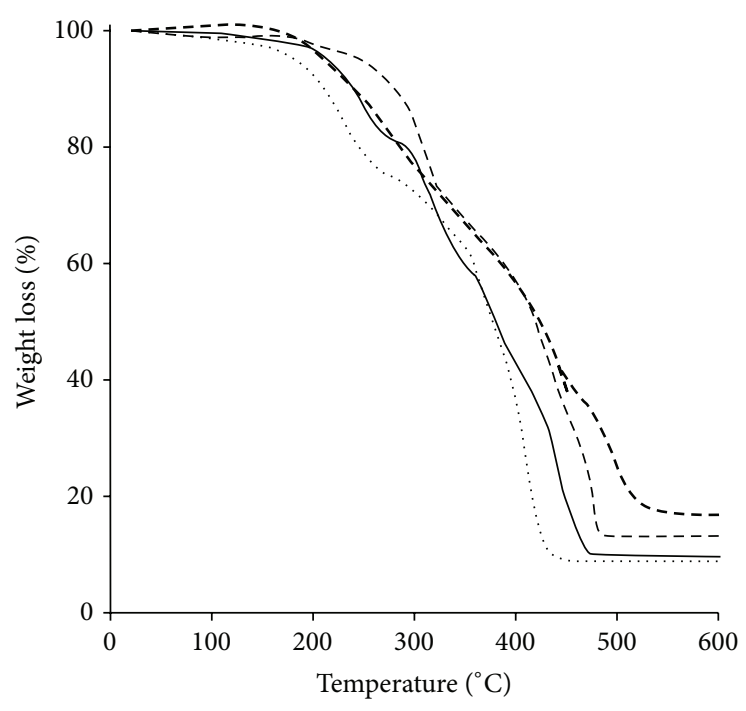

(b)

Figure 6: TGA curves of a mixed metal-oleate complex with $\mathrm{Mn}: \mathrm{Zn}$ ratios (a) $0.3: 0.7$ and (b) $0.7: 0.3$. The solid lines correspond to the mixed metal-oleate complex while the dotted line, small dashed line, and long dashed lines correspond to iron-, manganese-, and zinc-oleate complexes, respectively.

the $0.3: 0.7 \mathrm{Mn}: \mathrm{Zn}$ mixed metal-oleate complex. The nucleation stage still occurs at a relatively narrow temperature window, but there are two extra changes in weight loss rate seen that were absent in Figure 6(a). The first of the events occurs around $365^{\circ} \mathrm{C}$ and the second around $435^{\circ} \mathrm{C}$. The second is most likely due to a change in the rate of nanoparticle formation, as it is not very pronounced, but the first nicely lines up with the temperature required to begin nucleation in manganese-oleate complex. This allows for the eventual synthesis of nanoparticles at higher temperatures without the interference of poorly dissociated oleate ligands. In order to guarantee the synthesis of NPs with controlled composition, all of the different oleate complexes must be able to begin dissociation before nucleation or nanoparticle formation begins.

One potential way to remove this problem would be to create separate oleate complexes for each of the metals and then mix them during the thermal decomposition step. However, it has been shown that creating separate oleate complexes and then mixing them later for thermal decomposition leads to a broader size distribution and a decrease in shape control [35]. Another potential method is to use a different solvent with a higher boiling point to allow for the start of dissociation of all metal-oleate complexes present. This latter solution would ensure that each metal-oleate was involved in the burst nucleation and size focusing regimes [29]. Further development of scalable reactions needs to be conducted in order to facilitate the usage of $\mathrm{Mn}-\mathrm{Zn}$ ferrite nanoparticles as tracers.

\section{Conclusions}

Based upon the forgoing research we can make several conclusions as to the viability of scaling ternary ferrite NPs.
First, despite a range of synthetic strategies being applicable for nMag, they may not allow for complex mixed oxides to be prepared with the same elemental and size control. Second, a particular route may be successful for large-scale synthesis of one particular formula (i.e., $\mathrm{Mn}: \mathrm{Zn}=0.7: 0.3$ ), but at different compositional ratios NPs may be difficult to prepare due to the differences in the kinetics associated with the precursors with respect to the prenucleation, nucleation, and growth stages of NP formation. It is clear that a greater understanding is important before these types of material can be used on a large scale.

\section{Conflict of Interests}

The authors declare that there is no conflict of interests regarding the publication of this paper.

\section{Acknowledgments}

Financial support was provided by the Robert A. Welch Foundation (C-0002), nanoAlberta, part of Alberta Innovates Technology Futures, and Welsh Government Sêr Cymru Programme.

\section{References}

[1] M. Mahmoudi, P. Stroeve, A. S. Milani, and A. S. Arbab, Superparamagnetic Iron Oxide Nanoparticles: Synthesis, Surface Engineering, Cytotoxicity \& Biomedical Applications, Nanotechnology Science and Technology, Nova Science Publishers, Hauppauge, NY, USA, 2011.

[2] S. Gyergyek, D. Makovec, A. Kodre, I. Arčon, M. Jagodič, and M. Drofenik, "Influence of synthesis method on structural and magnetic properties of cobalt ferrite nanoparticles," Journal of Nanoparticle Research, vol. 12, no. 4, pp. 1263-1273, 2010. 
[3] D. T. Margulies, F. T. Parker, F. E. Spada et al., "Anomalous moment and anisotropy behavior in $\mathrm{Fe}_{3} \mathrm{O}_{4}$ films," Physical Review B, vol. 53, no. 14, pp. 9175-9187, 1996.

[4] S. P. Sena, R. A. Lindley, H. J. Blythe, C. Sauer, M. Al-Kafarji, and G. A. Gehring, "Investigation of magnetite thin films produced by pulsed laser deposition," Journal of Magnetism and Magnetic Materials, vol. 176, no. 2-3, pp. 111-126, 1997.

[5] D. Pan, A. Senpan, S. D. Caruthers et al., "Sensitive and efficient detection of thrombus with fibrin-specific manganese nanocolloids," Chemical Communications, no. 22, pp. 32343236, 2009.

[6] J. P. Jolivet and A. R. Barron, "Nanoparticle fabrication," in Environmental Nanotechnology: Applications and Impacts of Nanotechnology, M. R. Wiesner and J.-Y. Bottero, Eds., McGraw-Hill, New York, NY, USA, 2007.

[7] A. W. Orbaek, L. Morrow, S. J. Maguire-Boyle, and A. R. Barron, "Reagent control over the composition of mixed metal oxide nanoparticles," Journal of Experimental Nanoscience, vol. 10, no. 5, pp. 324-349, 2015.

[8] A. G. Roca, S. Veintemillas-Verdaguer, M. Port, C. Robic, C. J. Serna, and M. P. Morales, "Effect of nanoparticle and aggregate size on the relaxometric properties of MR contrast agents based on high quality magnetite nanoparticles," The Journal of Physical Chemistry B, vol. 113, no. 19, pp. 7033-7039, 2009.

[9] C. Sun, J. S. H. Lee, and M. Zhang, "Magnetic nanoparticles in MR imaging and drug delivery," Advanced Drug Delivery Reviews, vol. 60, no. 11, pp. 1252-1265, 2008.

[10] H. Lee, E. Lee, D. K. Kim, N. K. Jang, Y. Y. Jeong, and S. Jon, "Antibiofouling polymer-coated superparamagnetic iron oxide nanoparticles as potential magnetic resonance contrast agents for in vivo cancer imaging," Journal of the American Chemical Society, vol. 128, no. 22, pp. 7383-7389, 2006.

[11] L. Morrow, D. Potter, and A. R. Barron, "Detection of magnetic nanoparticles against proppant and shale reservoir rocks," Journal of Experimental Nanoscience, vol. 9, 2014.

[12] J. Park, K. An, Y. Hwang et al., "Ultra-large-scale syntheses of monodisperse nanocrystals," Nature Materials, vol. 3, no. 12, pp. 891-895, 2004.

[13] Y. Lee, J. Lee, C. J. Bae et al., "Large-scale synthesis of uniform and crystalline magnetite nanoparticles using reverse micelles as nanoreactors under reflux conditions," Advanced Functional Materials, vol. 15, no. 3, pp. 503-509, 2005.

[14] W. Cai and J. Wan, "Facile synthesis of superparamagnetic magnetite nanoparticles in liquid polyols," Journal of Colloid and Interface Science, vol. 305, no. 2, pp. 366-370, 2007.

[15] N. Bao, L. Shen, W. An, P. Padhan, C. H. Turner, and A. Gupta, "Formation mechanism and shape control of monodisperse magnetic $\mathrm{CoFe}_{2} \mathrm{O}_{4}$ nanocrystals," Chemistry of Materials, vol. 21, no. 14, pp. 3458-3468, 2009.

[16] B. D. Fahlman and A. R. Barron, "Substituent effects on the volatility of metal $\beta$-diketonates," Advanced Materials for Optics and Electronics, vol. 10, no. 3-5, pp. 223-232, 2000.

[17] Q. Song, Y. Ding, Z. L. Wang, and Z. J. Zhang, "Tuning the thermal stability of molecular precursors for the nonhydrolytic synthesis of magnetic $\mathrm{MnFe}_{2} \mathrm{O}_{4}$ spinel nanocrystals," Chemistry of Materials, vol. 19, no. 19, pp. 4633-4638, 2007.

[18] G. Rudolph and M. C. Henry, "The thermal decomposition of zinc acetylacetonate hydrate," Inorganic Chemistry, vol. 3, no. 9, pp. 1317-1318, 1964.

[19] C. Rath, S. Anand, R. P. Das et al., "Dependence on cation distribution of particle size, lattice parameter, and magnetic properties in nanosize $\mathrm{Mn}-\mathrm{Zn}$ ferrite," Journal of Applied Physics, vol. 91, no. 3, pp. 2211-2215, 2002.
[20] R. Arulmurugan, B. Jeyadevan, G. Vaidyanathan, and S. Sendhilnathan, "Effect of zinc substitution on Co-Zn and Mn-Zn ferrite nanoparticles prepared by co-precipitation," Journal of Magnetism and Magnetic Materials, vol. 288, no. 1, pp. 470-477, 2005.

[21] V. Šepelák, I. Bergmann, A. Feldhoff et al., "Nanocrystalline nickel ferrite, $\mathrm{NiFe}_{2} \mathrm{O}_{4}$ : mechanosynthesis, nonequilibrium cation distribution, canted spin arrangement, and magnetic behavior," Journal of Physical Chemistry C, vol. 111, no. 13, pp. 5026-5033, 2007.

[22] E. Manova, B. Kunev, D. Paneva et al., "Mechano-synthesis, characterization, and magnetic properties of nanoparticles of cobalt ferrite, $\mathrm{CoFe}_{2} \mathrm{O}_{4}$," Chemistry of Materials, vol. 16, no. 26, pp. 5689-5696, 2004.

[23] Z. H. Zhou, J. M. Xue, H. S. O. Chan, and J. Wang, "Transparent magnetic composites of $\mathrm{ZnFe}_{2} \mathrm{O}_{4}$ nanoparticles in silica," Journal of Applied Physics, vol. 90, no. 8, pp. 4169-4174, 2001.

[24] M. V. Kovalenko, M. I. Bodnarchuk, R. T. Lechner, G. Hesser, F. Schäffler, and W. Heiss, "Fatty acid salts as stabilizers in sizeand shape-controlled nanocrystal synthesis: the case of inverse spinel iron oxide," Journal of the American Chemical Society, vol. 129, no. 20, pp. 6352-6353, 2007.

[25] M. R. Buck, A. J. Biacchi, and R. E. Schaak, "Insights into the thermal decomposition of $\mathrm{Co}$ (II) oleate for the shapecontrolled synthesis of wurtzite-type CoO nanocrystals," Chemistry of Materials, vol. 26, no. 3, pp. 1492-1499, 2014.

[26] L. M. Bronstein, X. Huang, J. Retrum et al., "Influence of iron oleate complex structure on iron oxide nanoparticle formation," Chemistry of Materials, vol. 19, no. 15, pp. 3624-3632, 2007.

[27] Y. Chen, E. Johnson, and X. Peng, "Formation of monodisperse and shape-controlled $\mathrm{MnO}$ nanocrystals in non-injection synthesis: self-focusing via ripening," Journal of the American Chemical Society, vol. 129, no. 35, pp. 10937-10947, 2007.

[28] Y. Chen, M. Kim, G. Lian, M. B. Johnson, and X. Peng, "Side reactions in controlling the quality, yield, and stability of high quality colloidal nanocrystals," Journal of the American Chemical Society, vol. 127, no. 38, pp. 13331-13337, 2005.

[29] S. G. Kwon, Y. Piao, J. Park et al., "Kinetics of monodisperse iron oxide nanocrystal formation by 'heating-up' process," Journal of the American Chemical Society, vol. 129, no. 41, pp. 12571-12584, 2007.

[30] V. K. La Mer, "Nucleation in phase transitions," Industrial \& Engineering Chemistry, vol. 44, no. 6, pp. 1270-1277, 1952.

[31] N. T. N. Thanh, N. Maclean, and S. Mahiddine, "Mechanisms of nucleation and growth of nanoparticles in solution," Chemical Reviews, vol. 114, no. 15, pp. 7610-7630, 2014.

[32] C. S. Li, Y. N. Li, Y. L. Wu, B. S. Ong, and R. O. Loutfy, "Synthesis of zinc oxide nanocrystals by thermal decomposition of Zn-oleate in organic medium," Science in China Series E: Technological Sciences, vol. 51, no. 12, pp. 2075-2079, 2008.

[33] N. R. Jana, Y. Chen, and X. Peng, "Size- and shape-controlled magnetic ( $\mathrm{Cr}, \mathrm{Mn}, \mathrm{Fe}, \mathrm{Co}, \mathrm{Ni})$ oxide nanocrystals via a simple and general approach," Chemistry of Materials, vol. 16, no. 20, pp. 3931-3935, 2004.

[34] W. S. Chiu, P. S. Khiew, D. Isa et al., "Synthesis of twodimensional $\mathrm{ZnO}$ nanopellets by pyrolysis of zinc oleate," Chemical Engineering Journal, vol. 142, no. 3, pp. 337-343, 2008.

[35] N. Bao, L. Shen, Y. Wang, P. Padhan, and A. Gupta, "A facile thermolysis route to monodisperse ferrite nanocrystals," Journal of the American Chemical Society, vol. 129, no. 41, pp. 12374-12375, 2007. 

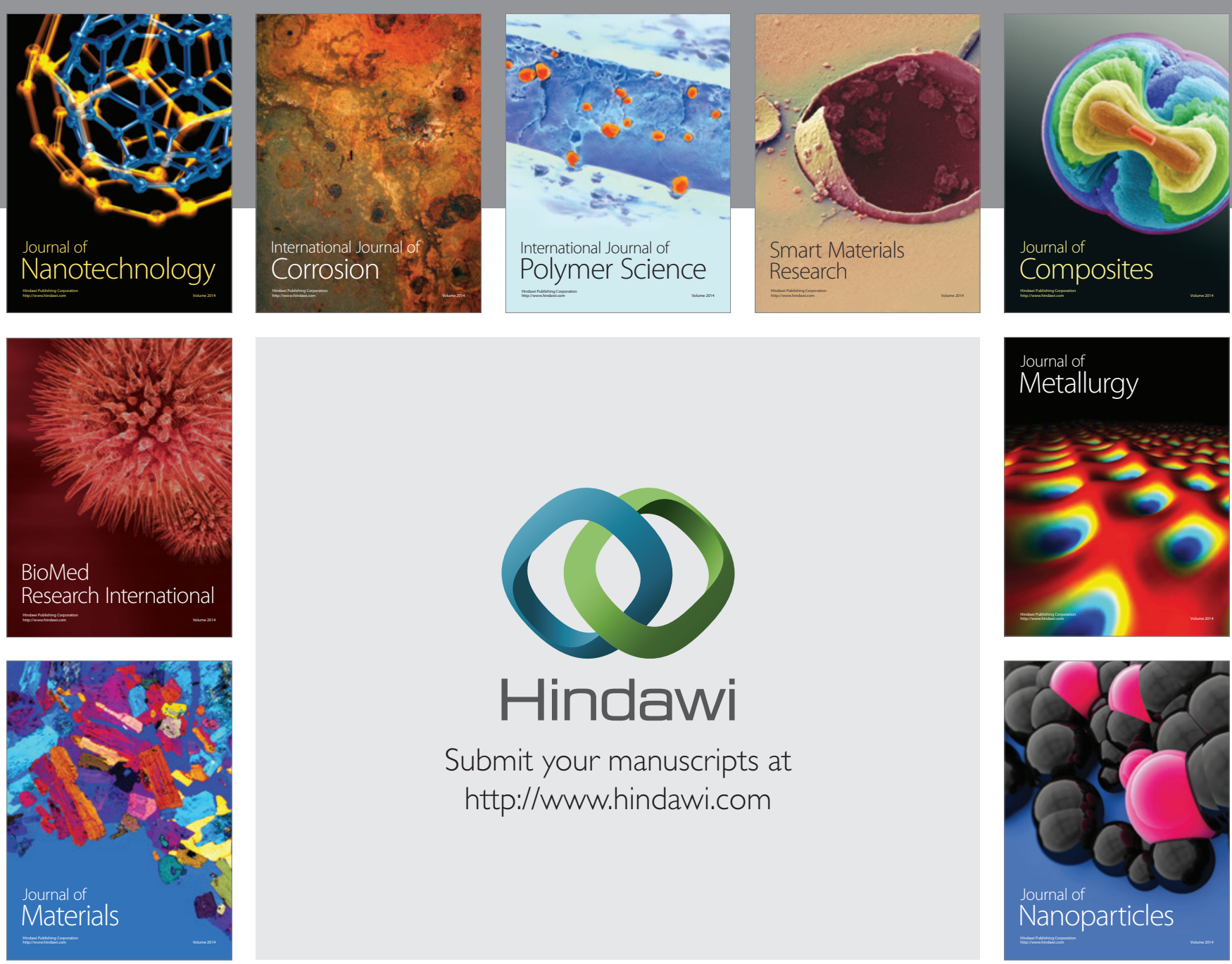

Submit your manuscripts at http://www.hindawi.com
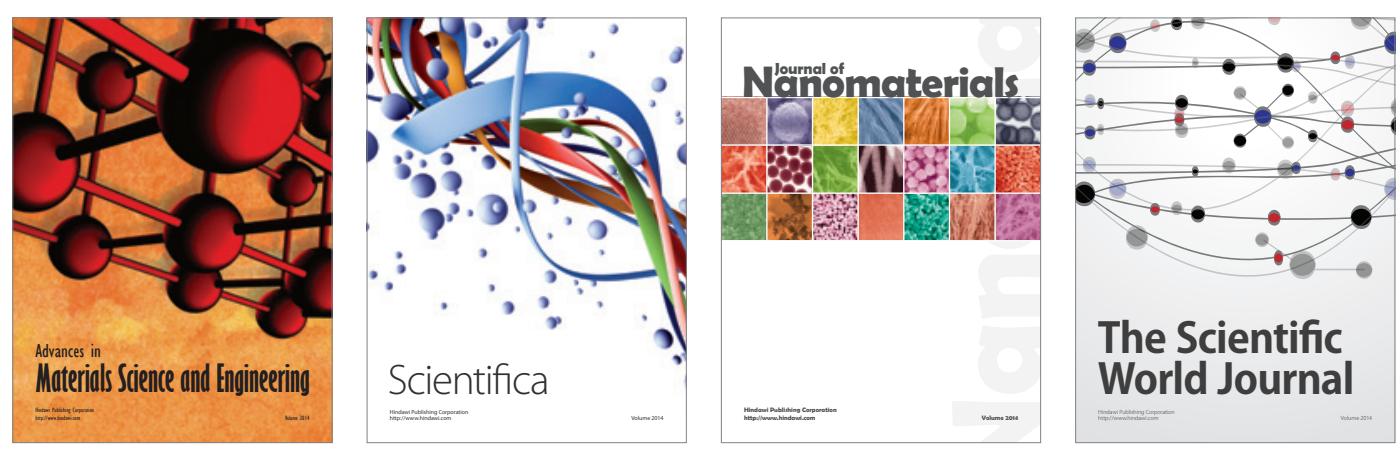

\section{The Scientific World Journal}
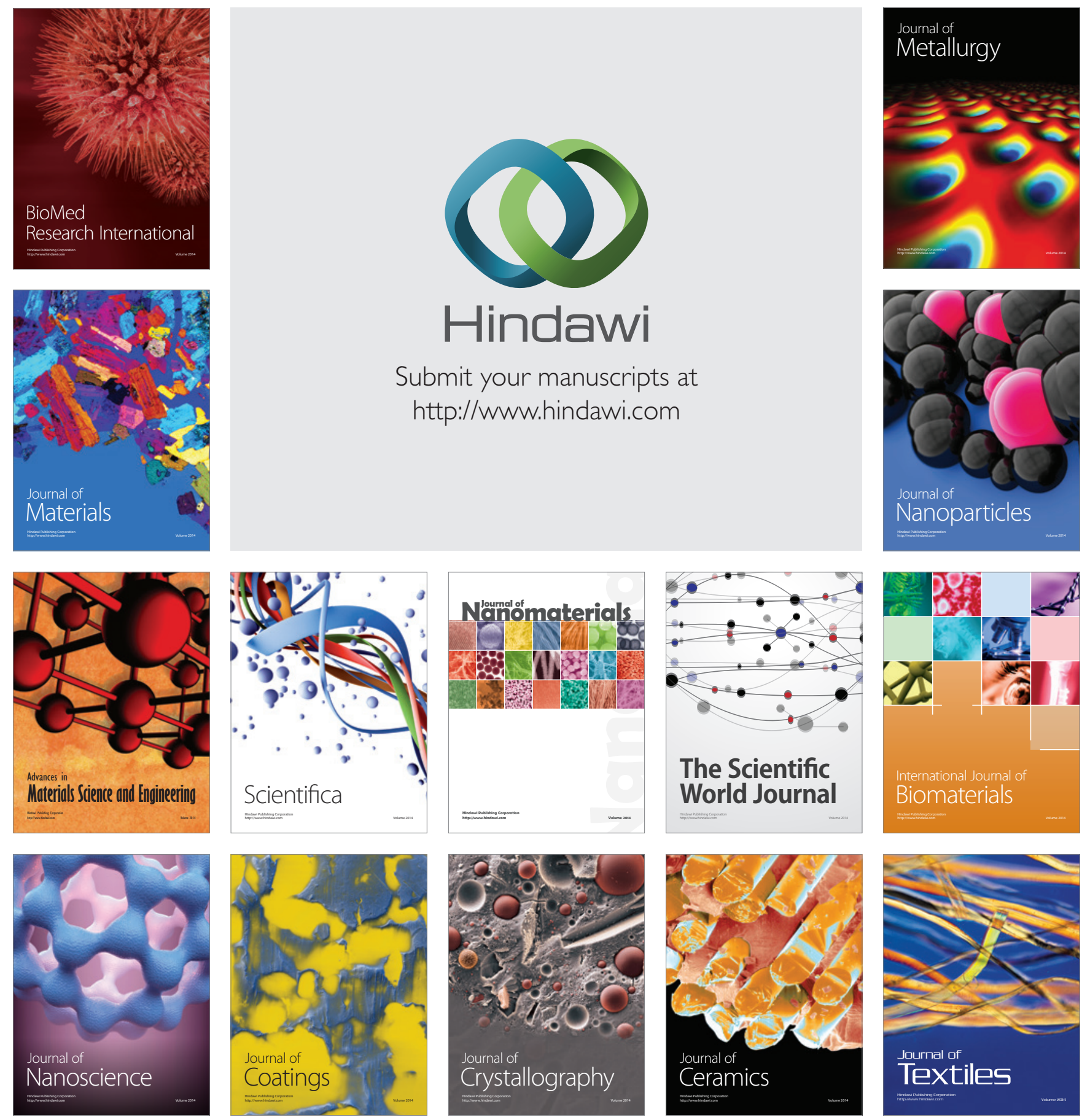